\title{
Multiple polymerase chain reaction for Microsporidium spp, Cryptosporidium spp, isospora belli and cyclospora cayetanensis parasites
}

\author{
Enedina Jiménez-Cardoso, Apolinar Cano-Estrada, Adrian-Cortes Campos, \\ Leticia Eligio-García \\ Laboratory of parasitology, children hospital of Mexico, Dr. Marquez 162. Colonia Doctores, Cuauhtémoc. Mexico DF \\ Email address: \\ Jimenezce@yahoo.com.mx (E. Jiménez-Cardoso)
}

\section{To cite this article:}

Enedina Jiménez-Cardoso, Apolinar Cano-Estrada, Adrian-Cortes Campos, Leticia Eligio-García. Multiple Polymerase Chain Reaction for Microsporidium spp, Cryptosporidium spp, Isospora Belli and Cyclospora Cayetanensis Parasites. American Journal of Bioscience and Bioengineering. Vol. 2, No. 2, 2014, pp. 33-36. doi: 10.11648/j.bio.20140202.12

\begin{abstract}
The purpose of this study was to establish the optimal conditions to performance a multiple Polymerase Chain Reaction (multiplex) to detect the presence of emerging parasites Cryptosporidium spp., Mycrosporidium spp., Isospora belli, and Cyclospora spp. simultaneously, by targeting the $16 \mathrm{~S}$ ribosomal gene in patients immunocompromised. After an exhaustive genetic analysis to obtain different primers sequences we tested the conditions of reagents concentration, time and temperature of reaction. The results showed the optimal conditions to make an efficient, specific, and reliable diagnostic method, performed in a simultaneous molecular reaction, to identify the presence of emerging parasites in faeces of patients susceptible to different infectious due to an immunocompromised status caused by diseases and chemotherapy to treat them. This method will help to decrease risk of complications and negative effects on life quality of ill patients.
\end{abstract}

Keywords: Cryptosporidium, Microsporidium, Isospora, Belli, Cyclospora, Multiplex, Immunocompromised, Emerging, Parasites

\section{Introduction}

The coccidia protozoan and Microsporidium spp are causes of diarrhea across the world; they cause traveler syndrome and epidemiological outbreak. Infection in immunocompetent people is silent but in patients with immunodeficiency it can be very serious. The term coccidia include Isospora belli, Cryptosporidium parvum, Cyclospora cayetanensis, of these parasites, the Cryptosporidium species are frequently detected in the clinical samples as well as Microsporidium spp [1].

Cryptosporidium spp have been recognized, that infect a wide variety of hosts, but Only two members of the Cryptosporidium genus, C. parvum and C. hominis, infect humans [2], these two species represent an important public health especially in immunocompromised individuals, infection of with these parasites is associated with a particularly high risk of fatality [3]. With the light microscope oocysts $\mathrm{C}$. parvum are observed as ovoid bodies sizing 2-4 $\mathrm{mm}$ diameter in samples of heces. There is currently no effective antimicrobial therapy for cryptosporidiosis [4].

The coccidian Isospora belli was renamed Cystoisospora belli in 2005, it rarely cause infection in immunocompetent humans, however, can be considered opportunistic intestinal pathogens in patients with HIV / AIDS in those who can produce a malabsorption syndrome with epigastric and eosinophilia epidemiologic studies have revealed that isosporiasis may be related to travel and immigration from Latin America [5]. This parasite causes a persistent watery diarrhea, malabsortion, and weight loss [6]. It is identified in diarrheal stool samples as characteristic oocysts, sporulated or not sporulated.

Cyclospora cayetanensis is a rapidly emerging parasite that has been implicated in both epidemic and endemic diarrheal illnesses around the world [7]. It has a worldwide distribution. Human infection causes a wide range of symptoms in immunocompetent patients, the most frequent 
being mild to severe diarrhea [8]. Cyclospora is endemic in tropical areas and particularly among immunocompromised individuals. The diagnosis is made by microscopic identification of the oocysts in feces. The recommended treatment for $\mathrm{C}$. cayetanensis is focused to avoid the symptoms and not the parasitic infection.

The Microspora genus is composed of a broad group of single-celled obligate intracellular protozoa that share a polar filament and are capable of infecting evolutionarilydistinct hosts, from insects and fish to birds and mammals [9]. Intestinal microsporidiasis is most frequently caused by the Encephalitozoon and Enterocytozoon species [10]. Diagnosis is difficult due to the size of spore is about $1 \mathrm{um}$ and Microsporidia consisting of several species with spores that vary in shape, size and method of cell division. When cellular immunity is very weak, some species produce severe disseminated disease.

Traditionally, diagnosis of these parasitic infections relies on microscopy-based observation and morphologybased identification of the organism in stool samples [11]. Alternative approaches have been recently developed, including immunological and molecular techniques based on advances in our knowledge of the physiological and genetic features of these emerging parasites [12]. Direct examination of fresh or concentrated feces remains the standard method of detection of I. belli and Giardia, since the large oocysts and cysts are readily visible by light microscopy without staining. However, microscopic identification of the smaller protozoan parasites is reliant upon the experience of the microscopist [13]. Since the morphological characteristics, size, and staining affinity are not uniform. Several studies have led to improved staining methods, and the newly developed molecular strategies have demonstrated high sensitivity and specificity for detecting and identifying the emerging human parasites, for this reason it is important establish a faster and efficient method of diagnosis.

The aim of this study was to establish the reaction conditions to detect the presence of Cryptosporidium spp., Microsporidium spp., Isospora belli, and Cyclospora cayetanensis simultaneously in fecal samples of patients with and without gastrointestinal symptomatology in an only single multiplex reaction.

\section{Materials and Methods}

\subsection{Parasites DNA and Fecal Specimens}

Positive DNA controls: DNA from Encephalitozoon hellem was extracted from ATCC $\AA 50451$ strain by Wizard $\AA$ genomic DNA purification kit (Promega. Madison, Wi. USA). Genomic DNA from Cryptosporidium parvum ATCC ${ }^{\circ}$ PRA-67D. Due to they are not commercially available cultures or genomic DNA of Isospora spp and Cyclospora spp, the oocysts were obtained from human fecal samples after the identification of parasitic forms under a light microscope by Faust
Method and stained with Lugol's iodine [14]. The parasitic structures were concentrated, purified and store to $4^{\circ} \mathrm{C}$ until their use, then DNA was extracted from both of them by use of the Fast DNA kit (MP Biomedicals, Oh, USA), according to the manufacturer's instructions. Obtained DNA was filtered through a QIAamp mini spin column (QIAGEN).

All DNA concentrations were measured by a spectrophotometer (Epoch; Biotek, Winooski, VT, USA) and used as control for multiple PCR.

\subsection{Primer Designing}

An exhaustive analysis of 1200 sequences of Microsporidium, 60 sequences of Isospora and an equal number of Cyclospora and Cryptosporidium spp were carried out using Mega 5.2 to make a multiple alignment to know similarities and dissimilarities and establish the more stable choice. The specificity was determined by the absence of dimerization with the Oligonalic software and Multiplex PCR was performed.

\subsection{Multiplex PCR Amplification}

Multiplex Reaction was performed by QuiagenR multiplex PCR plus Kit (Cat No. 206151) as directed by the instruction manual. The DNA samples were amplified in a thermocycler Axygen Maxygen and were visualized by agarose gel $1.5 \%$ stained with ethidium bromide.

We tested different concentrations of Magnesium, primers and DNA in a mixture reaction and a variation of cycles, time and temperature of the amplification program. The final conditions were: In a volume of $25 \mu \mathrm{L}$, consisting of $1 \times$ PCR master mix $0.2 \mathrm{uM}$ of primers reported in table 1, 1x Q solution, and 500ng of template DNA (DNA of Cryptosporidium spp., Microsporidium spp., Isospora belli, and Cyclospora cayetanensis in a mixture of equal concentration.). Thirty-five cycles of amplification were performed in a Maxi-gene thermal cycler (Axygen, Union City, CA, USA). After the initial denaturation of DNA at $95{ }^{\circ} \mathrm{C}$ for $5 \mathrm{~min}$, each cycle consisted of a denaturation step at $95{ }^{\circ} \mathrm{C}$ for 30 seconds, an annealing step at $58{ }^{\circ} \mathrm{C}$ for 45 seconds, and an extension step at $72{ }^{\circ} \mathrm{C}$ for 30 seconds. A final extension step was carried out at $68^{\circ} \mathrm{C}$ for $10 \mathrm{~min}$.

Table 1. Primers used in multiplex. A universal forward primer (UFP) and the specific reverse primer for each parasite were used. The theoretical melting temperature is showed.

\begin{tabular}{llll}
\hline Parasite & Primer & Sequence 5'-3' & Tm $\left({ }^{\circ} \mathbf{C}\right)$ \\
\hline UFP & PUF1 & ccggagagggagctg & 60.45 \\
Isospora & PibR & caccagaaggatgccgg & 61.23 \\
Microsporidium & PmiR & ctgcagcatccaccataga & 59.27 \\
Cryptosporidium & PcpR & catgctggagtattcaaggcat & 61.38 \\
Cyclospora & PccR & gcacaccctacgggcaag & 62.66 \\
\hline
\end{tabular}




\section{Results}

After some assays, we can obtain a multiband pattern with amplification fragments corresponding to Microsporidium spp. (198 bp), Cyclospora cayetanensis (295 bp), Isospora belli (324bp), and Cryptosporidium spp. (500 bp) (Figure 1).

We made a first assay with 35 amplification cycles, Tm $60^{\circ} \mathrm{C}, 300 \mathrm{ng}$ and $500 \mathrm{ng}$ of each DNA. The amplification could carry out with the higher concentration of DNA, but two bands sizing 300 and 400 bp were highlighted over the rest (data no shown).

The next assay was performance with 40 amplification cycles and $\mathrm{Tm}$ of $58^{\circ} \mathrm{C}, 500 \mathrm{ng}$ of DNA, $0.5 \mu \mathrm{M}$ primer mix and $0.2 \mu \mathrm{M}$ of primer mix. The resolution was improved with these conditions (data no shown).

The next assay was performance with 40 amplification cycles and $\mathrm{Tm}$ of $58^{\circ} \mathrm{C}, 500 \mathrm{ng}$ of DNA, $0.5 \mu \mathrm{M}$ primer mix and $0.2 \mu \mathrm{M}$ of primer mix. The resolution was improved with these conditions (data no shown).

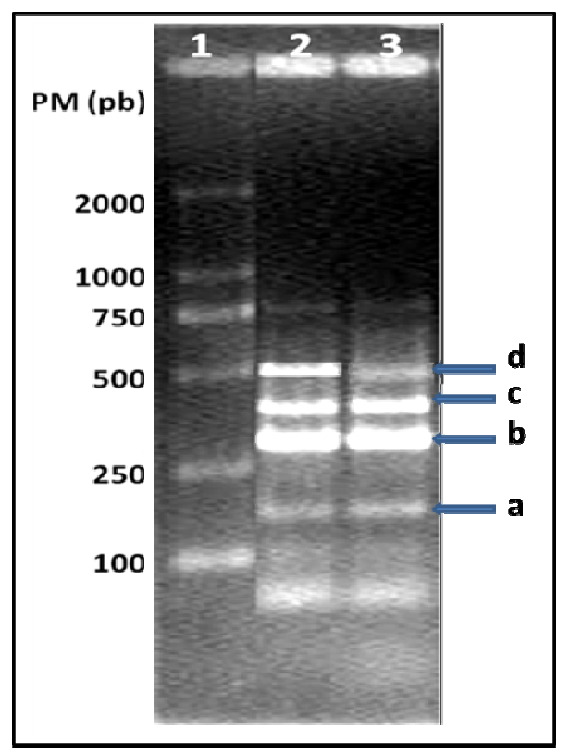

Figure 1. 1.5\% Agarose gel electrophoresis stained with ethidium bromide. Lane 1: PM ladder. Lane 2: amplification with $500 \mathrm{ng}$ of DNA and 0.5 $\mu M$ of primer mix. Lane 3: amplification with $500 \mathrm{ng}$ of DNA and $0.2 \mu \mathrm{M}$ of primer mix. All reactions were performed with 40 amplification cycles and $\mathrm{Tm} 58^{\circ} \mathrm{C}$. Arrows points to the amplified fragments corresponding to a) Microsporidium spp. (198 bp), b) Cyclospora cayetanensis (295 bp), c) Isospora belli (324bp), and d)Cryptosporidium spp. $(500 \mathrm{bp})$

The figure 1 shows two different assays with 40 amplification cycles and different concentrations of DNA (In a mixture of equal concentration of four parasites DNA) and primers shown in table 1 ; lane 2: 500 ng and $0.5 \mu \mathrm{M}$, lane 3: 500ng and $0.2 \mu \mathrm{M}$. We can observe the amplifications of DNA corresponding to four parasites.

\section{Discussion}

According to the current literature, the most routine method of diagnosis for the emerging parasites has been microscopic analysis with Faust staining, using three consecutive stool samples that have been concentrated. For Isospora and Giardia spp., this method is very effective, since the unsporulated oocyst stage of Cyclospora, which measures 8 to 10 micrometers, is found in the fecal smears of patients infected and the nucleated cysts of Giardia, varying from 10 to 15 micrometers are easily distinguishable by exclusively staining with iodine [15]. However, in the case of smaller parasites, such as Microsporidium, Cyclospora and Cryptosporidium spp., microscopic staining analyses are difficult and rely on the skill and experience of the investigator to achieve accurate diagnosis, In addition, the clinical importance of emerging parasites had been minimized, avoiding intentional search in microscopic analysis, this fact causes a high number of false negatives cases. Although staining methods increase the sensitivity and effectiveness of diagnosis, there are research studies that indicate that molecular methods increases from $65 \%$ to $97 \%$ of effectiveness, whit an analytical sensitivity at 50 to 500 oocysts $/ \mathrm{ml}$ of liquid stool to Cryptosporidium, from $54 \%$ until $67 \%$ of specificity with a sensitivity of 102 spores/g to Microsporidia and a sensitivity of 10 oocysts/g of feces for Cyclospora [16]. In the current study, we carried out FAUST coproparasitoscopic analysis to identify parasites in stool samples, and attempted to increase the diagnostic accuracy by performing staining with the Ziehl Neelsen method. Indeed, the number of positive cases was increased by the additional analysis. However, use of molecular PCR analysis identified even more positive cases, as well as providing sequence data that could help to identify the genotype of the parasite. During the course of the study, some samples were determined false negative, when the molecular study was carried out and the result was positive, the sample was concentrated and analyzed by light microscopy in a more directed way, in all cases samples were positives, although with a low number of parasitic structures. Molecular studies have the advantage of requiring a minimum amount of DNA to identify the presence of parasites in biological samples compared with microscopic methods, which are cheap and easy to make but difficult to interpret, which causes an underdiagnosis.

Molecular tools have proven useful in discovering and characterizing emerging parasites. There are many recent studies about implementing molecular diagnostic techniques based on PCR to generate knowledge on the epidemiology and treatment of these infections, but until today that is trying to establish a multiple PCR for identification of these organisms.

In a multiple PCR the objective is to amplify simultaneously in a one single tube different specific sequences and this to imply that the mixture of reagents and the amplification program be sufficient enough to allow the detection of each DNA sequence without inhibit the rest. First, it is necessary to choose or design oligonucleotides without interaction between them, having similar melting temperatures, specific for a single target 
DNA and that amplify sequences with different sizes to be resolved after amplification. Second it was necessary tested the concentrations of magnesium, DNA and primers to find the optimal concentrations of the reaction mixture.

Improved diagnostic molecular methods as multiplex for simultaneous identification of these parasites will feature higher specificity and sensitivity to facilitate more accurate and early diagnosis so that the appropriate therapeutic intervention may be initiated in a timely manner and this assay provides significant cost and labor savings for disease monitoring efforts in co-endemic locations.

\section{References}

[1] Botero, D., Restrepo, M., Parasitosis humanas a ed. CIB, Colombia.

[2] Flanigan, T., Whalen, C., Turner, J., .Cryptosporidium infection and CD count. Ann. Intern. Med.

[3] Gonçalves, E.M., Araújo, R.S., Orban, M., Matté, G.R., Matté, M.H., Corbett, C.E. Protocol for DNA extraction of Cryptosporidium spp. oocysts in fecal samples. Rev. Inst. Med. Trop. Sao Paulo

[4] Fayer, R., Morgan, U., Upton, S.J., Epidemiology of Cryptosporidium: transmission, detection and identification. Int. J. Parasitol.

[5] Bialek, R, Binder, N., Dietz, K., Knobloch, J., Zelck, U.E. Comparison of autofluorescence and iodine staining for detection of I. belli in feces. Am. J. Trop. Med. Hyg.

[6] Mirdha, B.R., Kabra, S.K., Samantray, J.C., Isosporiasis in children. Indian. Pediatric.

[7] Orlandi, P.A., Carter, L., Brinker, A.M., da Silva, A.J., Chu, D.M., Lampel, K.A., Monday, S.R., . Targeting singlenucleotide polymorphisms in the $\mathrm{S}$ rRNA gene to differentiate Cyclospora species from Eimeria species by multiplex PCR. Appl. Environ. Microbiol. , - .
[8] Weitz, J.C., Weitz, C.R., Canales, M.R., Moya, R.R., Cyclospora cayetanensis infection: updated review a propos of three cases of traveler's diarrhea. Rev. Chilena. Infectol.

[9] Wasson, K., Peper, R.L., Mammalian microsporidiosis. Vet. Pathol.

[10] Viriyavejakul, P., Nintasen, R., Punsawad, C., Chaisri, U., Punpoowong, B., Riganti, M., . High prevalence of Microsporidium infection in HIV-infected patients. Southeast. Asian. J.Trop. Med. Public. Health. , -

[11] Di Gliullo, A.B., Cribari, M.S., Bava, A.J., Cicconetti, J.S., Collazos, R., Cyclospora cayetanensis in sputum and stool samples. Rev. Inst. Med. Trop. Sao Paulo., ,-.

[12] Geurden, T., Berkvens, D., Casaert, S., Vercruysse, J., Claerebout, E. A Bayesian evaluation of three diagnostic assays for the detection of Giardia duodenalis in symptomatic and asymptomatic dogs. Vet. Parasitol.

[13] Coyle, C.M., Wittner, M., Kotler, D.P., Noyer, C., Orenstein, J.M., Tanowitz, H.B., Weiss, L.M., Prevalence of microsporidiosis due to Enterocytozoon bieneusi and Encephalitozoon (Septata) intestinalis among patients with AIDS-related diarrhea: determination by polymerase chain reaction to the microsporidian small-subunit rRNA gene. Clin. Infect. Dis.

[14] Faust, E.C., D'Antoni, J.S., Odom, V., Miller, M.J., Peres, C., Sawitz, W., Thomen, L.F., Tobie, L.E., Walker, J.H., A critical study of clinical laboratory techniques for the diagnosis of protozoan cysts and helminth eggs in feces. Am. J. Trop. Med.

[15] Visvesvara, G.S., Moura. H., Kovacs-Nace, E., Wallace, S., Eberhard, M.L., Uniform staining of Cyclospora oocysts in fecal smears by a modified safranin technique with microwave heating. J. Clin. Microbiol.

[16] Dong, J., Olano, J.P., McBride, J.W., Walker, D.H., Emerging pathogens: challenges and successes of molecular diagnostics. J. Mol. Diagn. 